\title{
Impact des Propriétés Mécaniques, Géométriques et thermiques des matériaux sur les Contraintes d'Interface, des structures renforcées par composites.
}

\author{
Bachir Kerboua ${ }^{1 . *}$, El Abbes Adda bedia ${ }^{2}$, Mohamed Kotbi ${ }^{3}$ \\ I l,2,3 Laboratoire des Matériaux \& Hydrologie, Université de Sidi Bel Abbés, Algérie. \\ kerbovac@yahoo.fr \\ * Correspondance, courriel :
}

\section{Résumé}

Actuellement, l'utilisation des plaques en composites, pour le renforcement des poutres et des structures métalliques, est une des méthodes récentes pour la réhabilitation des structures qui présentent un vieillissement ou des défauts de forme ou de chargement. Ces méthodes permettent, ainsi, de prolonger la durée de vie de ces structures sous un coût d'exploitation réduit et, assurent un minimum de pollution à l'environnement. Dans cet article une analyse originale, par une modélisation analytique des contraintes d'interface entre la poutre et la plaque de renforcement en (FRP) a été finalisée.

L'originalité dans le modèle adopté et développé, met en évidence une théorie qui tient compte, en plus de l'effet thermique, des effets de déformations des adhérents sous la force longitudinale, chose qui n'a pas été prise en compte par les études précédentes. Ce phénomène de déformation des adhérents, donne une approche plus probable sur les contraintes d'interface des structures renforcées, il est appelé effet "I shear lag II. La résolution numérique montre que la contrainte de cisaillement et la contrainte normale sont significatives et, sont concentrées à l'extrémité de la plaque de renforcement, phénomène appelé effet de bord (edges effects). Une étude paramétrique et physique des adhérents a été effectuée pour montrer leur influence sur la valeur des contraintes.

Mots-clés : Renforcement, effets de décollement, Effet thermique, contraintes interfaciales. 


\section{Abstract \\ Impact of the Mechanical properties and Geometrical and thermal of material on interfacial stresses, of the structures strengthened by composites}

Currently, the use of the composites plates, for the reinforcement of the beams and the metal structures, is one of the recent methods for the rehabilitation of the structures presenting a failure or defects of form or loading. These methods make it possible, thus, to prolong the lifespan of the structures under a reduced cost of exploitation and, ensure a minimum of pollution of the environment.

The original study in this paper carried out an analytical solution to estimate shear and peel-off stresses, as, interfacial stress analysis concentration under the thermal load and shear lag deformation. The theoretical prediction is compared with author solutions from numerous researches. This phenomenon of deformation of the members, which gives probably approach on the study of interface of the reinforced structures, is called "shear lag effect". The resolution in this paper shows that the shear stress and the normal stress are significant and, are concentrated at the end of the plate of reinforcement, called "edge effect". A parametric study is carried out to show the effects of the variables of design and the physical properties of materials.

\section{Keywords : Retrofitted structures; peel off effect; interfacial stresses; thermal effect}

\section{Introduction}

La méthode de renforcement par composites a été utilisée avec sucée pour la réparation des structures métalliques durant de nombreuses années [1-7]. Dans le domaine de génie civil, le renforcement par RFP composites a été bien utilisé pour le renforcement et la réparation des structures (poutres en acier ou en béton), chose qui intéresse de plus en plus les chercheurs actuellement.

Dans les structures, le joint constitut le point faible pour tout mode de défaillance. Dans le but de faire face à ce problème, la connaissance des contraintes de distribution au niveau du joint adhésif est importante. Au niveau de ce joint, la zone d'interface entre les structures et l'adhésive comporte une concentration de contraintes, notamment à l'extrémité de la structure et de la plaque en composite, à cause de la discontinuité géométrique dans cette zone [2-7]. Goland et Reissner [4] ont mené une classique analyse sur les contraintes de distribution au niveau du joint adhésive. Hart-Smith [5] a 
proposé une procédure d'analyse et un critère de dégradation avec une formulation de Goland et Reissner [4].

La distribution de la contrainte d'interface des composés, poutre chargée en flexion et plaque soumise à la traction est la simulation de toute étude de concentration de contrainte à l'extrémité libre des composants structure et composite. Malek et Saadatmanesh, Smith et Teng $[6,7]$ ont mis en revue une approximation des solutions pour les contraintes interfaciales développées dans la littérature, et ont présenté une nouvelle solution tenant en compte des termes négligés par les études précédentes, au niveau de la poutre et de la plaque.

Quantrill et Al. [8] ont mené une performance étude expérimentale et analytique pour quantifier le transfert de charge entre la poutre et la plaque composite. La solution analytique prédit la distribution de la contrainte longitudinale le long de la plaque RFP et montre que $98 \%$ environ de la force de collage est localisée sur les $100 \mathrm{~mm}$ à partir de l'extrémité de la plaque. Un autre problème qui se présente de façon très précis dans les poutres en acier et qui n'a pas été soulevé par les études précédentes c'est la différence de valeurs des coefficients de dilatation des deux composés. Le coefficient typique pour les poutres en acier est de $10.2 \times 10^{-6}$ et celui pour la plaque en composite est de valeur nul. Denton mène une analyse des contraintes d'interface, de cisaillement et normal au niveau de la couche de l'adhésif résultant de la différence des coefficients de dilatation des deux substrats, et montre que les contraintes d'interface causant des débuts de fissuration se localisent au environ de la différence de $50^{\circ} \mathrm{C}$ de température.

Comme les autres solutions développées, ce travail présente dans ce papier une formulation qui détermine les contraintes d'interfaces (contrainte de cisaillement et contrainte normale dans l'interface adhésive en tenant compte non seulement de la charge thermique mais également de l'effet II shear lag ॥ qui, donne plus de précision sur le comportement de la poutre et de la plaque composite, chose qui a été négligé par les études précédentes. Les résultats soulèvent que, les différentes méthodes ont été mis à l'évidence pour valider la comparaison et la précision de l'étude, en incluant l'influence de la forme géométrique et les propriétés des matériaux montrent bien l'impact de cette modélisation.

\section{Fondements théoriques}

La figure $I$ montre la forme géométrique de la structure renforcée avec ses paramètres dimensionnels, ainsi, que la section selon la coupe A-A pour représenter la configuration de l'interface du joint adhésif. Dans notre étude et d'analyse des contraintes d'interface, on va prendre des hypothèses de base [6-11]. 
1) On suppose que tous les matériaux sont linéaires et élastiques

2) La poutre est simplement supportée selon une section plane

3) La contrainte de cisaillement et la contrainte normale au niveau du joint adhésif sont supposées invariables selon la couche adhésive.

4) Les contraintes de déformations au niveau de la poutre et de la plaque composite sont prises en considération.

5) Le moment de déformation de la couche d'adhésive est négligé.
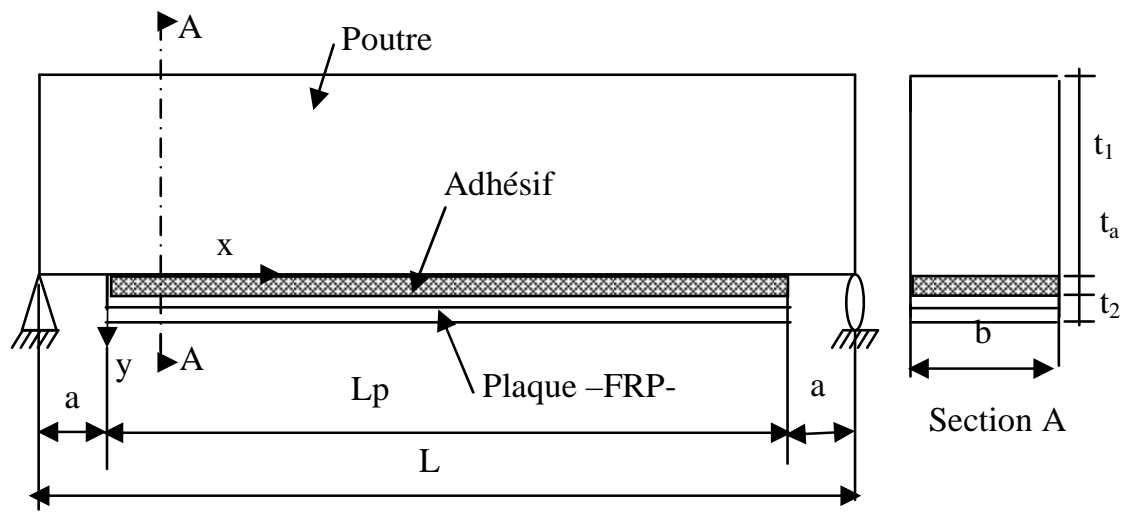

Figure 1 : Géométrie de la structure

\section{2-2. Distribution des efforts dans une section de la structure renforcée.}

La figure 2, montre une section de la poutre et de la plaque en FRP. Dans cette figure, V, $M, N$ sont l'effort de cisaillement, le moment de flexion et la force longitudinale, respectivement, $\mathrm{T}$ et $\sigma$ sont la contrainte de cisaillement et la contrainte normale à l'interface, ainsi, $\dagger$ c'est la composant de l'épaisseur. Les annotations $b$, $a$, et $p$ représentent la poutre, l'adhésif et la plaque, ce sont les annotations que l'on va utiliser dans ce travail. A partir de la troisième hypothèse, la force de cisaillement et la tension longitudinale dans l'adhésif sont négligées. 




Figure 2: Elément infinitésimal de la structure

L'équilibre longitudinal de l'élément de la plaque FRP selon l'axe $x$, donne [6-11].

$$
\frac{d N_{2}(x)}{d x}=-\tau(x) b_{2}
$$

L'équilibre transversal de l'élément de la plaque FRP selon l'axe y, donne

$$
\frac{d V_{2}(x)}{d x}=-\sigma(x) b_{2}
$$

L'équilibre de la poutre en acier dans le sens de $\mathrm{x}$ et dans le sens de $\mathrm{y}$, donne.

$$
\begin{gathered}
\frac{d N_{1}(x)}{d x}=-\tau(x) b 1 \\
\frac{d V_{1}(x)}{d x}=+\sigma(x) b_{1}-q
\end{gathered}
$$

L'équilibre des forces et des moments dans la direction longitudinale donne les équations 5 et 6.

$$
\frac{d M_{1}(x)}{d x}=V_{1}(x)-\tau(x) b_{2} y_{1}
$$




$$
\frac{d M_{2}(x)}{d x}=V_{2}(x)-\tau(x) b_{2} y_{2}
$$

\section{2-3. Distribution de la contrainte de cisaillement.}

La déformation de l'adhésif s'écrit:

$$
\gamma=\frac{d u(x, y)}{d y}+\frac{d v(x, y)}{d x}
$$

$u(x, y)$ ef $v(x, y)$ sont les déplacement longitudinal et transversal.

La contrainte de cisaillement s'écrit:

$$
\tau=G a .\left(\frac{d u(x, y)}{d y}+\frac{d v(x, y)}{d x}\right)
$$

La dérivée de l'équation 8 donne

$$
\frac{d \tau}{d x}=G a \cdot\left(\frac{d^{2} u(x, y)}{d y d x}+\frac{d^{2} v(x, y)}{d x d y}\right)
$$

Selon la troisième $\left(3^{\text {eme }}\right)$ hypothèse, la contrainte de cisaillement ne varie pas dans le sens de l'épaisseur de l'adhésive, et la déformation varie de façon linéaire, ce qui peut simplifier l'équation 9.

$$
\frac{d^{2} u}{d y d x}=\frac{1}{t a} \cdot\left(\frac{d u_{1}}{d x}+\frac{d u_{2}}{d x}\right)
$$

$u_{1}$ ef $u_{2}$ sont les déplacements dans le sens longitudinal à la base de la poutre et au sommet de la plaque FRP.

Leur dérivée selon l'axe des $x$ donne $[7,8,9]$.

$$
\begin{aligned}
& \frac{d u_{1}}{d x}=\varepsilon_{1}(x)=\alpha_{1} \Delta T+\frac{M_{1} t_{1} / 2}{E_{1} I_{1}}+\frac{N_{1}}{E_{1} A_{1}}-\frac{t_{1}}{3 G_{1}} \cdot \frac{d \tau(x)}{d x} \\
& \frac{d u_{2}}{d x}=\varepsilon_{2}(x)=\alpha_{2} \Delta T-\frac{M_{2} t_{2} / 2}{E_{2} I_{2}}+\frac{N_{2}}{E_{2} A_{2}}-\frac{t_{2}}{3 G_{2}} \cdot \frac{d \tau(x)}{d x}
\end{aligned}
$$

Avec $\mathcal{E}$, la déformation, $\alpha$ le coefficient de dilatation thermique, $\Delta T$ l'écart de température, $E$, I et $A$ le module d'élasticité, le moment quadratique de la section des éléments substrats et la section des éléments.

L'équilibre des moments dans la structure renforcée donne l'équation 13:

$$
M_{T}(x)=M_{1}(x)+M_{2}(x)+N(x)\left[y_{1}+y_{2}+t_{a}\right]
$$


$M_{T}(x)$, est le moment total appliqué sur la structure Le moment dans chaque adhèrent sera :

$$
\begin{gathered}
M_{1}(x)=\frac{R}{R+1}\left[M_{T}(x)-b_{2} \int_{0}^{x} \tau(x)\left(y_{1}+y_{2}+t_{a}\right) d x\right] \\
M_{2}(x)=\frac{1}{R+1}\left[M_{T}(x)-b_{2} \int_{0}^{x} \tau(x)\left(y_{1}+y_{2}+t_{a}\right) d x\right]
\end{gathered}
$$

La dérivée des équations 14 et 15 donne.

$$
\begin{aligned}
& \frac{d M_{1}(x)}{d x}=\frac{R}{R+1}\left[V_{T}(x)-b_{2} \tau(x)\left(y_{1}+y_{2}+t_{a}\right)\right] \\
& \frac{d M_{2}(x)}{d x}=\frac{1}{R+1}\left[V_{T}(x)-b_{2} \tau(x)\left(y_{1}+y_{2}+t_{a}\right)\right]
\end{aligned}
$$

On remplaçant la dérivée des équations 11 et 12 dans la dérivée de l'équation 9, on aura l'expression de l'équation de la contrainte tangentielle.

$$
\frac{d^{2} \tau(x)}{d x^{2}}=\frac{G 0}{t_{a}}\left(\begin{array}{l}
\frac{d M_{1}(x)}{d x} \frac{t_{1} / 2}{E_{1} I_{1}}+\frac{d M_{2}(x)}{d x} \frac{t_{2} / 2}{E_{2} I_{2}}+\frac{d N(x)}{d x} \frac{1}{E_{1} A_{1}} \\
-\frac{d N_{2}(x)}{d x} \frac{1}{E_{2} A_{2}}-\frac{d^{2} \tau(x)}{d x^{2}}\left(\frac{t_{1}}{3 G_{1}}+\frac{t_{2}}{3 G_{2}}\right)
\end{array}\right)
$$

On remplace chaque terme des équations $1,3,5$ et 6, dans l'équation 18 de la dérivée de la contrainte de cisaillement, on aura l'équation 19 qui, est l'équation finale de la contrainte de cisaillement :

avec

$$
\begin{gathered}
\frac{d^{2} \tau(x)}{d x^{2}}-K_{1} b_{2}\left(\frac{\left(y_{1}+y_{2}\right)\left(y_{1}+y_{2}+t_{a}\right)}{E_{1} I_{1}+E_{2} I_{2}}+\frac{1}{E_{1} A_{1}}+\frac{1}{E_{2} A_{2}}\right) \tau(x) \\
+K_{1}\left(\frac{y_{1}+y_{2}}{E_{1} I_{1}+E_{2} I_{2}}\right) V_{T}(x)=0 \\
K_{1}=\frac{1}{\left(\frac{t_{a}}{G_{a}}+\frac{t_{2}}{3 G_{2}}+\frac{t_{1}}{3 G_{1}}\right)}
\end{gathered}
$$

La solution générale de l'équation 19 de la contrainte de cisaillement est indiquée par l'équation 21.

$$
\tau(x)=B_{1} \cosh (\lambda x)+B_{2} \sinh (\lambda x)+m_{1} V_{T}(x)
$$




$$
\begin{gathered}
\lambda^{2}=K_{1} b_{2}\left(\frac{\left(y_{1}+y_{2}\right)\left(y_{1}+y_{2}+t_{a}\right)}{E_{1} I_{1}+E_{2} I_{2}}+\frac{1}{E_{1} A_{1}}+\frac{1}{E_{2} A_{2}}\right) \\
m_{1}=\frac{K_{1}}{\lambda^{2}}\left(\frac{y_{1}+y_{2}}{E_{1} I_{1}+E_{2} I_{2}}\right)
\end{gathered}
$$

$\mathrm{B}_{1}$ ef $\mathrm{B}_{2}$, sont des constantes déterminées par les conditions aux limites. Dans cette étude la poutre soumise à un chargement reparti, avec déformation de la structure et intégration de la charge thermique.

$$
B_{1}=-\frac{K_{1}}{\lambda}\left[\frac{y_{1}}{E_{1} I_{1}} M_{T}(0)+\left(\alpha_{1}-\alpha_{2}\right) \Delta T\right]+\frac{m_{1} q}{\lambda}=-B_{2}
$$

\section{2-4. Distribution de la contrainte normale.}

La contrainte normale dans l'adhésive peut être exprimée comme suit :

$$
\sigma_{n}(x)=K_{n} \Delta w(x)=K_{n}\left[w_{2}(x)-w_{1}(x)\right]
$$

Où $K n$ est la rigidité normale de l'adhésive par unité de longueur et peut déduite comme suit :

$w_{1}(x)$ ef $w_{2}(x)$ sont les déplacements verticales de l'adhérent 1 et 2 respectivement. En dérivant l'équation 25 deux fois, il en résulte :

$$
\frac{d^{2} \sigma_{n}(x)}{d x^{2}}=K_{a}\left[\frac{d^{2} w_{2}(x)}{d x^{2}}-\frac{d^{2} w_{1}(x)}{d x^{2}}\right]
$$

En considérant les relations, moment - courbure de la poutre et la plaque de renforcement on aura les équations 27 et 28 :

$$
\begin{gathered}
\frac{d^{2} w_{1}(x)}{d x^{2}}=-\frac{M_{1}(x)}{E_{1} I_{1}} \\
\frac{d^{2} w_{2}(x)}{d x^{2}}=-\frac{M_{2}(x)}{E_{2} I_{2}}
\end{gathered}
$$

L'équilibre de l'adhérent 1 et 2 mène aux relations suivantes des moments et des charges de cisaillement $[1,5,7]$ : 


$$
\begin{array}{r}
\frac{d M_{1}(x)}{d x}=V_{1}(x)-b_{2} y_{1} \tau(x) \\
\frac{d M_{2}(x)}{d x}=V_{2}(x)-b_{2} y_{2} \tau(x)
\end{array}
$$

La substitution des dérivées des équations 29 et 30 dans la quatrième dérivée de la contrainte normale obtenue par l'équation 26, on aura l'équation différentielle régissant la contrainte normale de l'interface :

$$
\begin{aligned}
& \frac{d^{4} \sigma_{n}(x)}{d x^{4}}+\frac{E_{a} b_{2}}{t_{a}}\left(\frac{1}{E_{1} I_{1}}+\frac{1}{E_{2} I_{2}}\right) \sigma_{n}(x)+ \\
& \frac{E_{a} b_{2}}{t_{a}}\left(\frac{y_{1}}{E_{1} I_{1}}-\frac{y_{2}}{E_{2} I_{2}}\right) \frac{d \tau(x)}{d x}+\frac{q E_{a}}{t_{a} E_{1} I_{1}}=0
\end{aligned}
$$

La solution générale de l'équation 31 est :

$$
\begin{aligned}
& \sigma_{n}(x)=e^{-\beta x}\left[C_{1} \cos (\beta x)+C_{2} \sin (\beta x)\right]+e^{\beta x}\left[C_{3} \cos (\beta x)+C_{4} \sin (\beta x)\right] \\
& -n_{1} \frac{d \tau(x)}{d x}-n_{2} q
\end{aligned}
$$

Pour des larges valeurs de $x$, on suppose que la contrainte normale tend vers zéro, et il en résulte $C_{3}=C_{4}=0$. La solution générale devient :

$$
\begin{gathered}
\sigma_{n}(x)=e^{-\beta x}\left[C_{1} \cos (\beta x)+C_{2} \sin (\beta x)\right]-n_{1} \frac{d \tau(x)}{d x}-n_{2} q \\
\beta=\sqrt[4]{\frac{E_{a} b_{2}}{4 t_{a}}\left(\frac{1}{E_{1} I_{1}}+\frac{1}{E_{2} I_{2}}\right)} \\
n_{1}=\left(\frac{y_{1} E_{2} I_{2}-y_{2} E_{1} I_{1}}{E_{1} I_{1}+E_{2} I_{2}}\right) ; \quad n_{2}=\frac{E_{2} I_{2}}{b_{2}\left(E_{1} I_{1}+E_{2} I_{2}\right)}
\end{gathered}
$$

$C_{1}$ et $C_{2}$, sont les coefficients constants d'intégration qui seront obtenus par les conditions aux limites.

$$
\begin{gathered}
C_{1}=\frac{E_{a}}{2 \beta^{3} t_{a}} \frac{1}{E_{1} I_{1}}\left[V_{T}(0)+\beta M_{T}(0)\right]-\frac{n_{3}}{2 \beta^{3}} \tau(0)+ \\
\frac{n_{1}}{2 \beta^{3}}\left(\left.\frac{d^{4} \tau(x)}{d x^{4}}\right|_{x=0}+\left.\beta \frac{d^{3} \tau(x)}{d x^{3}}\right|_{x=0}\right)
\end{gathered}
$$

et 
avec

$$
\begin{gathered}
C_{2}=-\frac{E_{a}}{2 \beta^{2} t_{a}} \frac{1}{E I_{1}} M_{T}(0)-\left.\frac{n_{1}}{2 \beta^{2}} \frac{d^{3} \tau(x)}{d x^{3}}\right|_{x=0} \\
n_{3}=\frac{E_{a} b_{2}}{t_{a}}\left(\frac{y_{1}}{E_{1} I_{1}}-\frac{y_{2}}{E_{2} I_{2}}\right)
\end{gathered}
$$

\section{Etude paramétrique et interprétation}

Un programme machine basé sur un modèle numérique Matlab a été établi dans ce sens et, selon les équations mentionnées ci-dessus afin de déterminer les contraintes à l'interface de la structure renforcée par plaque composites FRP, avec les conditions et les hypothèses décrites précédemment.

La longueur de la poutre $L=(3000 \mathrm{~mm})$, la largeur $b l=b 2=(200 \mathrm{~mm})$, l'épaisseur de la poutre $\mathrm{tl}=(300 \mathrm{~mm})$, l'épaisseur de la plaque $t 2=(4 \mathrm{~mm})$, l'épaisseur de l'adhésif $t a=$ $(2 \mathrm{~mm})$, le module d'élasticité de la poutre $\mathrm{El}=(30000 \mathrm{MPa})$, le module d'élasticité de la plaque FRP E2 $=(100000 \mathrm{MPa})$, le module de cisaillement de l'adhésif $\mathrm{Ga}=(740 \mathrm{MPa})$, le coefficient de poisson de la poutre, la plaque et de l'adhésif $\mathrm{Nu}=(0.35)$, la longueur libre $a=(300 \mathrm{~mm})$, la longueur de l'adhésive $L p=(2400 \mathrm{~mm})$.

\section{3-1. Résultats et discussion}

Plusieurs paramètres influent sur la valeur des contraintes d'interface, comme c'est montré dans les Figures 3, 4, 5, 6, et 7. Les propriétés des matériaux, l'épaisseur de la plaque, l'épaisseur de l'adhésive et la longueur de la plaque par rapport à l'extrémité libre, sont les facteurs qui influent sur les valeurs de ces contraintes qui, varient de 1.48 à $7.4 \mathrm{MPa}$ pour la contrainte normale et varient de 2.74 à $13.2 \mathrm{MPa}$ pour la contrainte de cisaillement. Le cas le plus frappant c'est la variation de ces contraintes, qui est proportionnelle à l'épaisseur de la plaque et au module d'élasticité de la plaque et inversement proportionnelle à l'épaisseur de l'adhésive, Figures 5, 6,7.

Un maximum de la valeur des contraintes est à l'extrémité de la plaque de renforcement, c'est un phénomène appelé effet de bord, qui est le signe des premières défaillances de la structure. Cet effet de bord est accentué par II l'effet thermique II et "I'effet shear lag $\|$ étudiés dans cette recherche comme une originalité et définies par le stage l et le stage $1+2$.

* Présent travail Stage I, on a tenu compte des paramètres pris par Smith et Teng, qui, ont donné un maximum de la contrainte normale de $1.48 \mathrm{MPa}$ et, un maximum de la 
contrainte de cisaillement de $2.74 \mathrm{MPa}$, plus le facteur thermique, qui donne un maximum de la contrainte normale de $4.4 \mathrm{MPa}$ et, un maximum de la contrainte de cisaillement de $8.4 \mathrm{MPa}$, Figures 3 et 4.

*Présent travail Stage $1+2$, on a tenu compte des paramètres pris par Smith et Teng, qui, ont donné un maximum de la contrainte normale del.48 $\mathrm{MPa}$ et, un maximum de la contrainte de cisaillement de $2.74 \mathrm{MPa}$, plus les deux effets II thermique et shear lag 1 couplés, qui donnent un maximum de la contrainte normale de $7.4 \mathrm{MPa}$ et un maximum de la contrainte de cisaillement de $13.2 \mathrm{MPa}$, Figures 3 et 4.

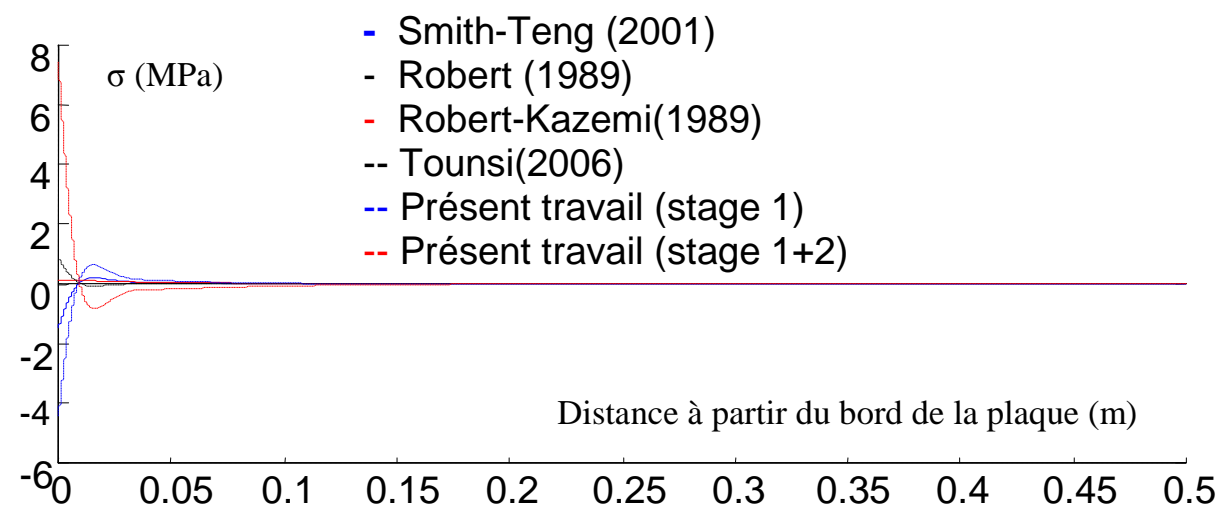

Figure 3: Variation de la contrainte normale pour différentes théories de renforcement des structures

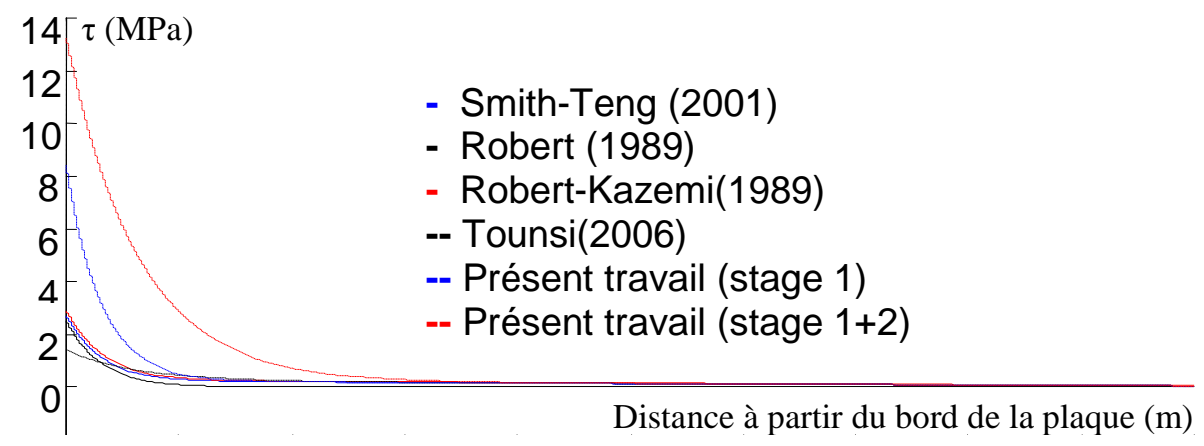

Figure 4: Variation de la contrainte tangentielle pour différentes théories de renforcement des structures 


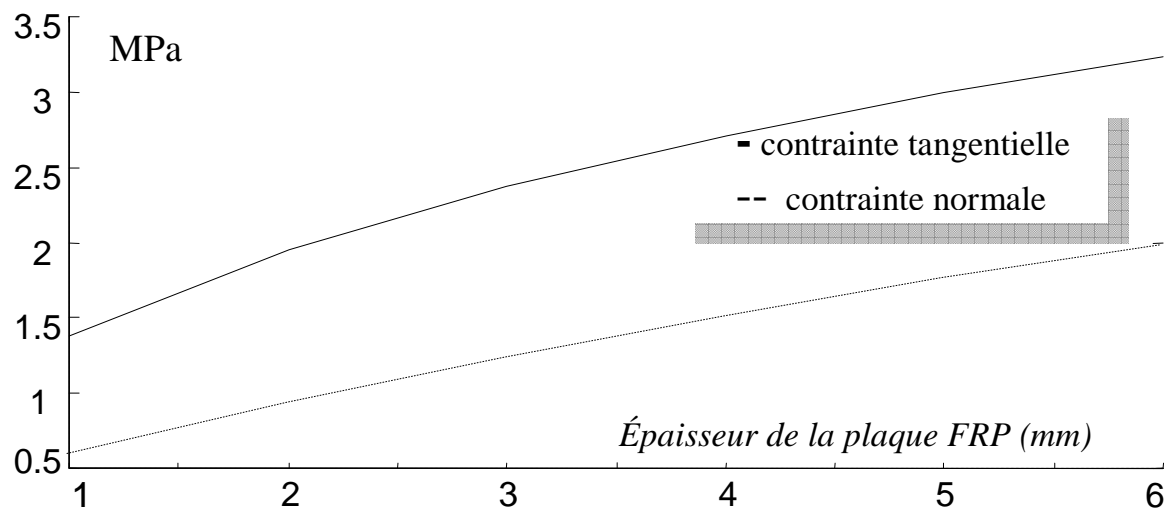

Figure 5: Variation du maximum des contraintes en fonction de l'épaisseur de la plaque composites-FRP-

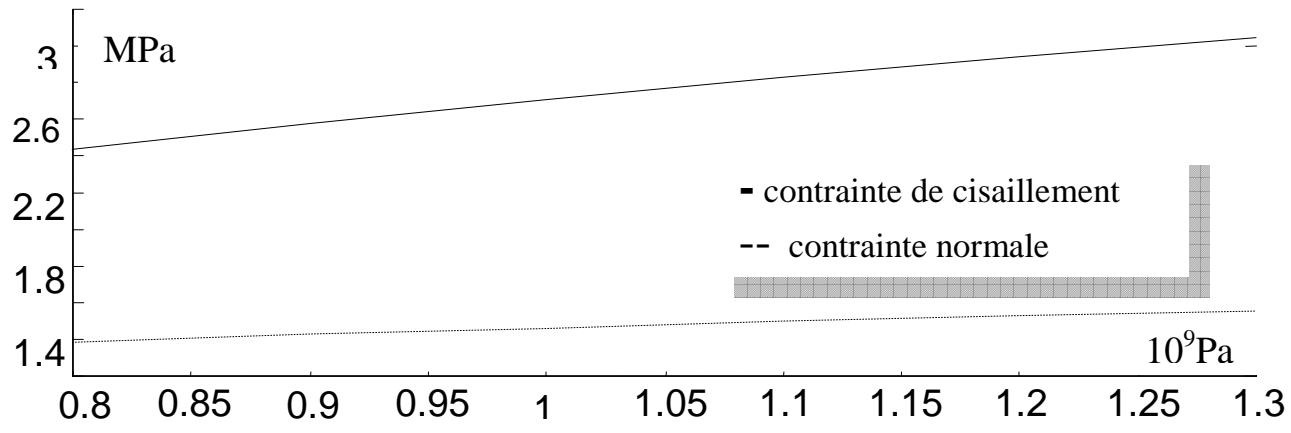

Figure 6: Variation du maximum des contraintes en fonction module de cisaillement de l'adhésive-Ea-

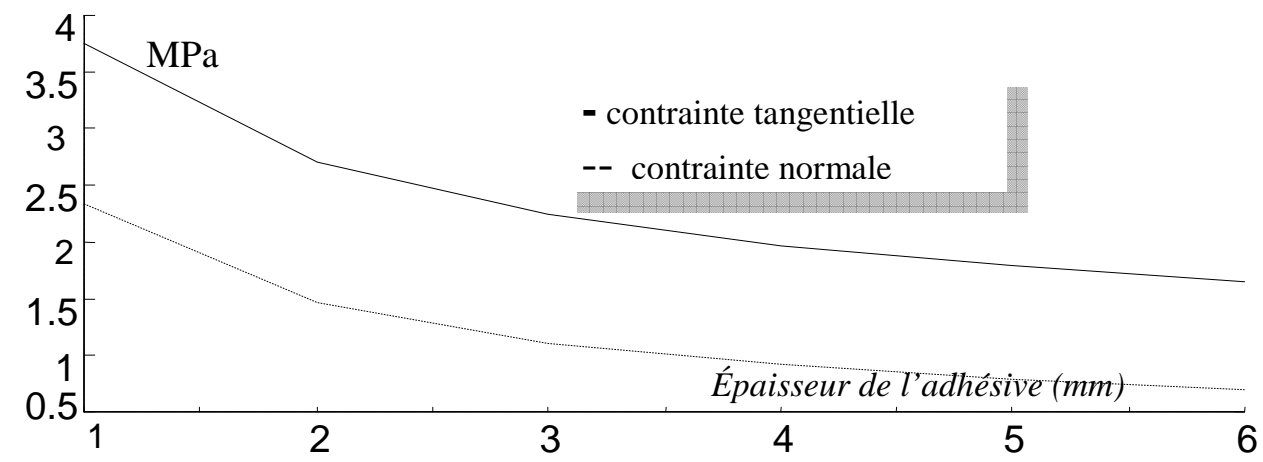

Figure7 : Variation du maximum des contraintes en fonction de l'épaisseur de l'adhésive-ta- 


\section{Conclusion}

Ce travail de recherche a été basé sur l'approche originale concernant l'analyse de la concentration des contraintes d'interface entre la poutre et la plaque en composite FRP, chose qui a été simulée selon le modèle examiné et déduites par les équations formulées. L'approche a été définie selon un modèle élastique, considérant la linéarité de cisaillement à travers l'épaisseur de l'adhésif et tenant en compte la déformation des adhérents dô à l'effort de cisaillement. La théorie présentée tient compte du modèle thermique dô à la différence des coefficients de dilatation, dans l'analyse des structures renforcées. Cette charge thermique montre bien l'effet de la concentration de contrainte à l'interface, notamment, sous la déformation des éléments du à l'effort de cisaillement, appelé effet shear lag. L'étude paramétrique montre que le maximum de la contrainte de cisaillement et de la contrainte normal est à l'extrémité des adhérents. Cette concentration est proportionnelle l'épaisseur de l'adhésive et inversement proportionnelle au module d'élasticité de la plaque et à l'épaisseur de la plaque composites. On note que l'effet II shear lag II prouve son importance sur la contrainte de cisaillement et l'effet de décollement de ces structures renforcées, notamment avec la présence de l'effet thermique. Dans le prochain article, on va entamer une approche qui va tenir compte de "l'effet taper II, utilisé comme réducteur de la concentration des contraintes à l'interface poutre-plaque composites (FRP).

\section{Références}

[1] - A. Tounsi, "Improved theoretical solution for interfacial stresses in concrete beams strengthened with FRP plate", Int. J. of Solids and Structure v.43, (2006), pp. $4154-4174$.

[2] - T. M. Roberts, "Approximate analysis of shear and normal stress concentrations in adhesive layer of plated RC beams", The Structure Engineering, London, v.67, no.12, (1989), pp. $229-233$.

[3] - A. Tounsi and S. Benyoucef, "Interfacial stresses in externally FRP-plated concrete beams", Int. J. of Adhesion and Adhesives, v.95, (2007), pp207-215.

[4] - M. Goland, and E. Reissner, J. APPL. mECH. V.11, (1994), pp.11-27.

[5] - L.J. Hart-Smith, "Adhesive bonded single lap joint", Douglas Air- Craft co., NASA, (1973), Langley Contract Report CR-112236.

[6] - M. Malek, H. Saadatmanesh and M.R. Ehsani, "Prediction of failure load of R/C 
beams strengthened with FRP plate due to stress concentration at the plate end", ACl Structural Journal, v.95, no.2, (1998), ppl $42-152$

[7] - S.T. Smith, and J.G. Teng, "Interfacial stresses in plated RC beams", Engineering Structures, v.23, no.7, (2001), pp857-871.

[8] - R. J. Quantrill, L. C. Hollaway and AM. Thorne, "Experimental and Analytical investigations of FRP-strengthened beam response: Part I", Mag Concrete Res, v.48, no.117, (1996), pp331 - 342.

[9] - O. Rabinovich, and Y. Frostig, "Closed-from high order analysis of RC beams strengthened with FRP" Journal of Composites for Construction, ASCE, v.4, (2000), pp65 - 74 .

[10] - H. S. Shen, J. G. Teng and J. Yang, "Interfacial stresses in beams and slabs bonded with a thin plate", Journal of Engineering Mechanics, ASCE, v.127, no.4, (2001), pp399-406.

[11] - 0. Vilnay, "The analysis of reinforced concrete beams strengthened by epoxy bonded steel plates", Int. Journal of Cement Composites Lightweight Concrete, v.10, no.2, (1988), pp73-78. 